\title{
CISTICERCOSE INTRAMEDULAR
}

\author{
RELATO DE CASO
}

\section{FERNANDO LUIZ ROLEMBERG DANTAS* ${ }^{*}$ WALTER JOSÉ FAGUNDES-PEREYRA**, CLÁUDIA TAVARES DE SOUZA***, MARCELO GABRIEL VEGA****, ATOS ALVES DE SOUZA*}

\begin{abstract}
RESUMO - A cisticercose é a doença parasitária que mais comumente envolve o sistema nervoso central. O acometimento espinhal varia de $0,7 \%$ a 5,8\%, sendo que a forma intramedular é uma manifestação rara. Relatamos um caso de cisticercose intramedular ao nível de C4-C5, em paciente de 52 anos, feminina, atendida no serviço de Neurologia e Neurocirurgia da Santa Casa de Belo Horizonte. Foi submetida a tratamento cirúrgico para exérese da lesão, com boa evolução pós-operatória e recuperação progressiva dos déficits. Concluimos que apesar de patologia rara (foram encontrados 45 casos após revisão da literatura), deve entrar no diagnóstico diferencial de lesões medulares, especialmente em pacientes com história de neurocisticercose e naqueles que vivem ou procedem de regiões onde é a doença é endêmica.
\end{abstract}

PALAVRAS-CHAVE: cisticercose, medula espinhal.

\section{Intramedular cysticercosis: case report}

ABSTRACT - Cysticercosis is the most ordinary parasitary disease involving the nervous system. The involvement of the spine is rare, ranging from $0.7 \%$ to $5.8 \%$, and the intramedullary incidence is rather uncommon. We report the case of a 52-year-old female patient with intramedullary cysticercosis at the C4-C5 level, treated at the Neurology and Neurosurgery Service of Santa Casa de Belo Horizonte. The patient was operated with the complete exeresis of the lesion and had a good outcome. Forty-five cases of intramedullary cysticercosis were found in the literature review. We conclude that although it is a rare pathology, intramedullary cysticercosis should be included in the differential diagnosis of intramedullary lesions, mainly in cases of patients with previous diagnosis for neurocysticercosis and also of those who live in endemic areas.

KEY WORDS: spinal cord, cysticercosis.

A cisticercose (CI) é a doença parasitária que mais envolve o sistema nervoso central (SNC). O acometimento espinhal ocorre em $0,7 \%$ a 5,8\% dos pacientes, sendo que a forma intramedular é uma rara manifestação $0^{1-5}$. Apesar de frequentemente estar associada à lesões cerebrais pode apresentarse isoladamente ${ }^{1}$. Pode ser classificada em: vertebral, extradural, leptomeníngea (subaracnóidea) e intramedular ${ }^{2}$, sendo a extradural de ocorrência excepcional, e as formas leptomeníngeas seis a oito vezes mais comuns que as intramedulares ${ }^{3}$.

Tendo em vista a raridade dessa patologia, decidimos pelo relato do presente caso, analisando aspectos patogênicos, clínicos, radiológicos e terapêuticos da doença, através de revisão da literatura.

Santa Casa de Belo Horizonte: *Neurocirurgião da Santa Casa de Belo Horizonte e Professor da Faculdade de Ciências Médicas de Minas Gerais (FCMMG); **Residente de Neurocirurgia da Santa Casa de Belo Horizonte; ***Neurocirurgiã do Hospital Municipal Odilon Behrens; ****Neurologista da Santa Casa de Belo Horizonte, Professor da FCMMG e Chefe do Serviço de Neurologia do Hospital Municipal Odilon Behrens. Aceite: 14janeiro-1999.

Dr. Fernando Luiz Rolemberg Dantas - Rua Ceará 565, 5ªndar - 30150-310 Belo Horizonte MG - Brasil. FAX 0312263281. 


\section{RELATO DO CASO}

MPS, 52 anos, sexo feminino, feoderma, lavradora, encaminhada ao serviço de Neurologia e Neurocirurgia da Santa Casa de Belo Horizonte com história de em julho-1997 ter começado a apresentar cefaléia holocraniana, de forte intensidade, associada a náuseas e vômitos, tendo sido submetida à tomografia computadorizada de crânio (TCC) que evidenciou dilatação moderada do sistema ventricular, com calcificações milimétricas nas regiões frontal, parietal e cápsula interna à esquerda, tendo sido diagnosticado neurocisticercose com hidrocefalia (Fig 1). Foi então optado pela colocação de derivação ventrículo-peritoneal (Hospital Santa Paula), com melhora do estado geral. Após três meses iniciou a apresentar diminuição de força no dimidio esquerdo, progressiva, atingindo também o dimidio direito, associada a parestesias tipo formigamento e diminuição da sensibilidade à esquerda. Ao exame físico apresentava-se com tetraparesia desproporcional, maior à esquerda, no membro inferior esquerdo, grau I, com tetra-hiperreflexia, sem sinal de Hoffman, com sinal de Babinski à esquerda. Sem alteração de sensibilidade. À ressonância nuclear magnética (RNM) da medula evidenciou-se lesão hipointensa em região cervical alta (C4-C5), intramedular (Fig 2).

Foi submetida a hemilaminectomia esquerda, em C3-T1, para exérese da lesão, intramedular, ao nível de C4-C5, que apresentava consistência amolecida e membrana translúcida, característica de cisticerco. O estudo histológico confirmou tratar-se de cisticerco (Fig 3).

No pós-operatório, a paciente evoluiu bem com melhora discreta dos déficits motores e das parestesias. Após um ano da cirurgia, a paciente apresentava melhora significativa da força, apesar de ainda manter discreta tetraparesia espástica.

\section{DISCUSSÃO}

Segundo Trelles, foi Paranoli, em 1550, quem primeiro descreveu a neurocisticercose na espécie humana. Entretanto, atribui-se a Rumler (1558) a primeira descrição precisa das doença ${ }^{6}$. A primeira referência a cisticercose no canal raquídeo é atribuída a Rokitanski, em 1856.

Durante o ciclo biológico da Taenia solium o homem pode tornar-se hospedeiro acidental de

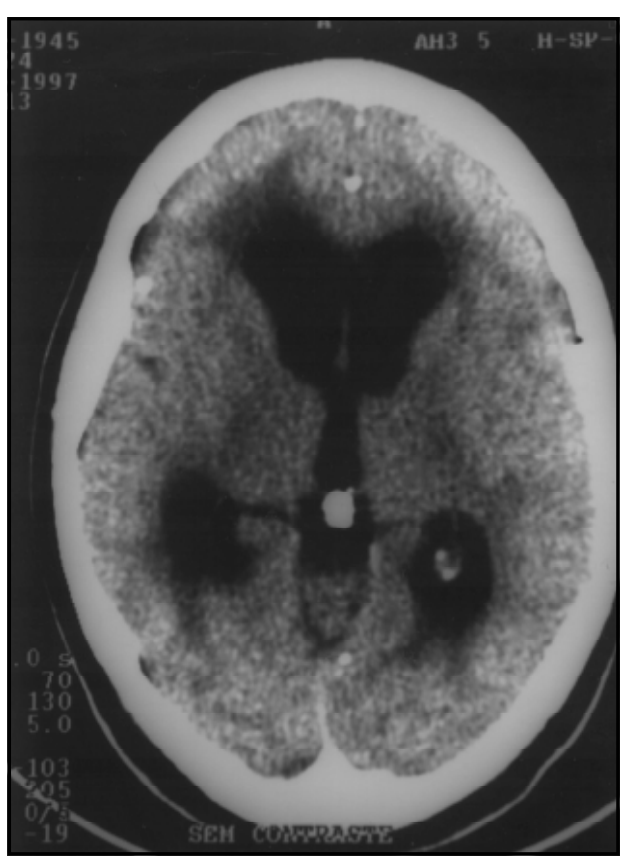

Fig 1. Tomografia computadorizada de crânio evidenciando hidrocefalia moderada com calcificações intraparenquimatosas. sua forma larvária. Seja pela ingestão ou por auto-infestação interna, os ovos produzidos pelo verme adulto alcançam o estômago, onde se rompem pela ação do suco gástrico. Os embriões são então liberados e, penetrando na parede do intestino delgado, alcançam a circulação. O embrião vai se alojar preferencialmente no tecido neural, no tecido subcutâneo, no músculo esquelético e no globo ocular, onde se desenvolve na forma larvária o Cysticercus cellulosae $e^{3}$.

Até 1992 havia 97 casos de cisticercose espinhal descritos na literatura mundial, sendo apenas 33 intramedulares ${ }^{1-3,5}$. Atualmente são 45 os casos de cisticercose intramedular (Tabela $1)^{2}$. Parece haver uma proporcionalidade entre o fluxo sanguíneo relativo a cada região da medula e a ocorrência de cisticercose intramedular ${ }^{1,3,7}$. Assim, predomina a localização torácica $(55,1 \%)$, seguida pela cervical $(24,1 \%)$ e finalmente pela lombossacra $(20,6 \%)^{3}$. A migração da larva inferiormente ao cérebro, para o espaço subaracnoideano ${ }^{2}$ e a via hematogênica venosa $^{5}$ são hipóteses que tentam explicar a forma leptomeníngea. A disseminação hemato- 


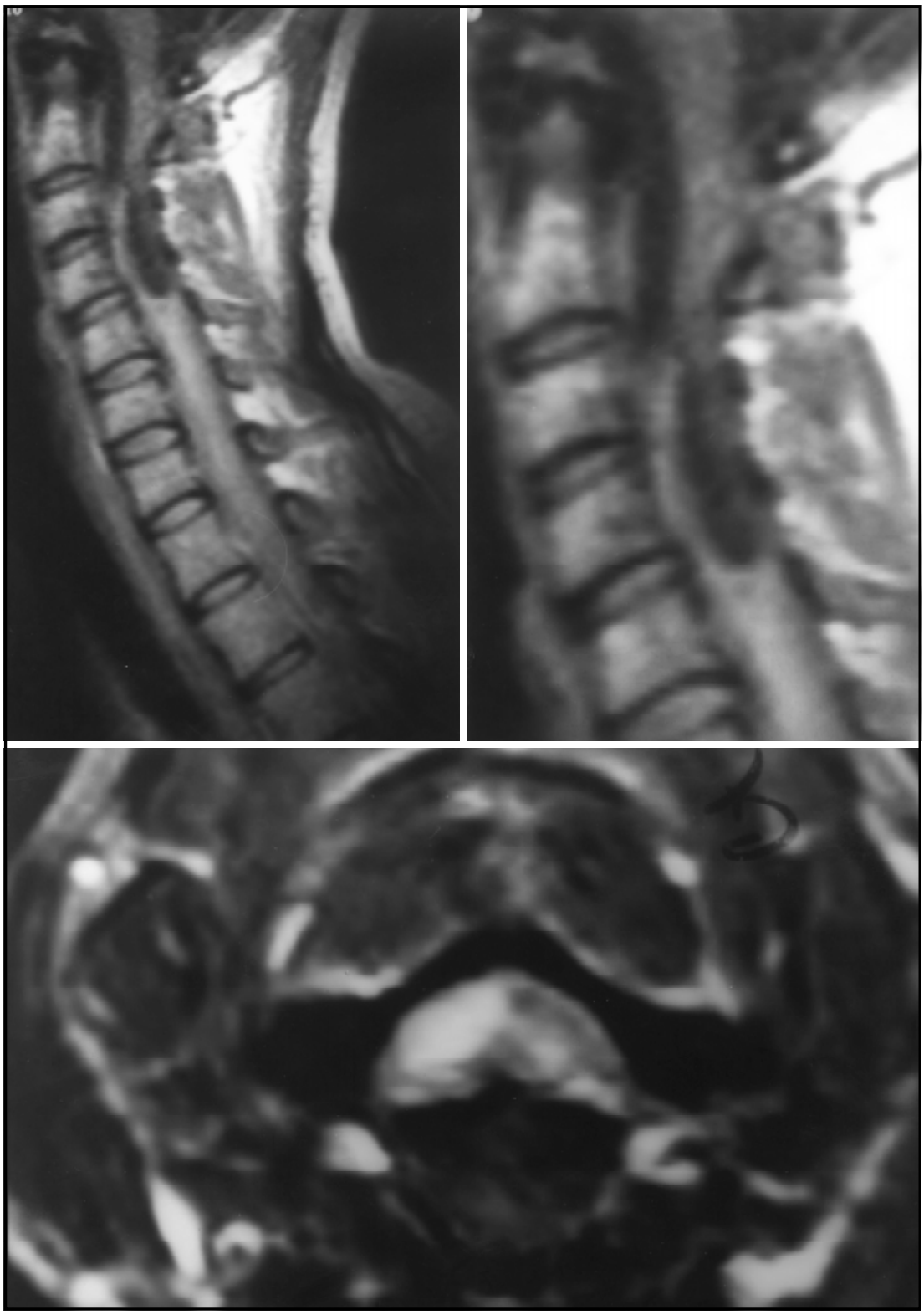

Fig 2. Ressonância nuclear magnética da medula. Em T1, evidencia-se lesão intramedular, hipointensa, ao nível de C4-C5, em cima. Corte transversal, em C5, evidenciando lesão intramedular, em baixo.

gênica e a via ventriculo-ependimária têm sido postuladas como meios da larva atingir o interior da medula $^{1-3,5}$. A teoria hematogênica é a mais atraente pois, sendo os cisticercos distribuídos no hospedeiro pela corrente circulatória, esta levaria os parasitas ao tecido medular³. Já a via ventrículo-ependimária não é muito clara, pois se o parasito descesse pelo canal central da medula seria mais provável que a maioria das vesículas fosse encontrada em nível cervical e não predominantemente torácico, como ocorre na realidade. 


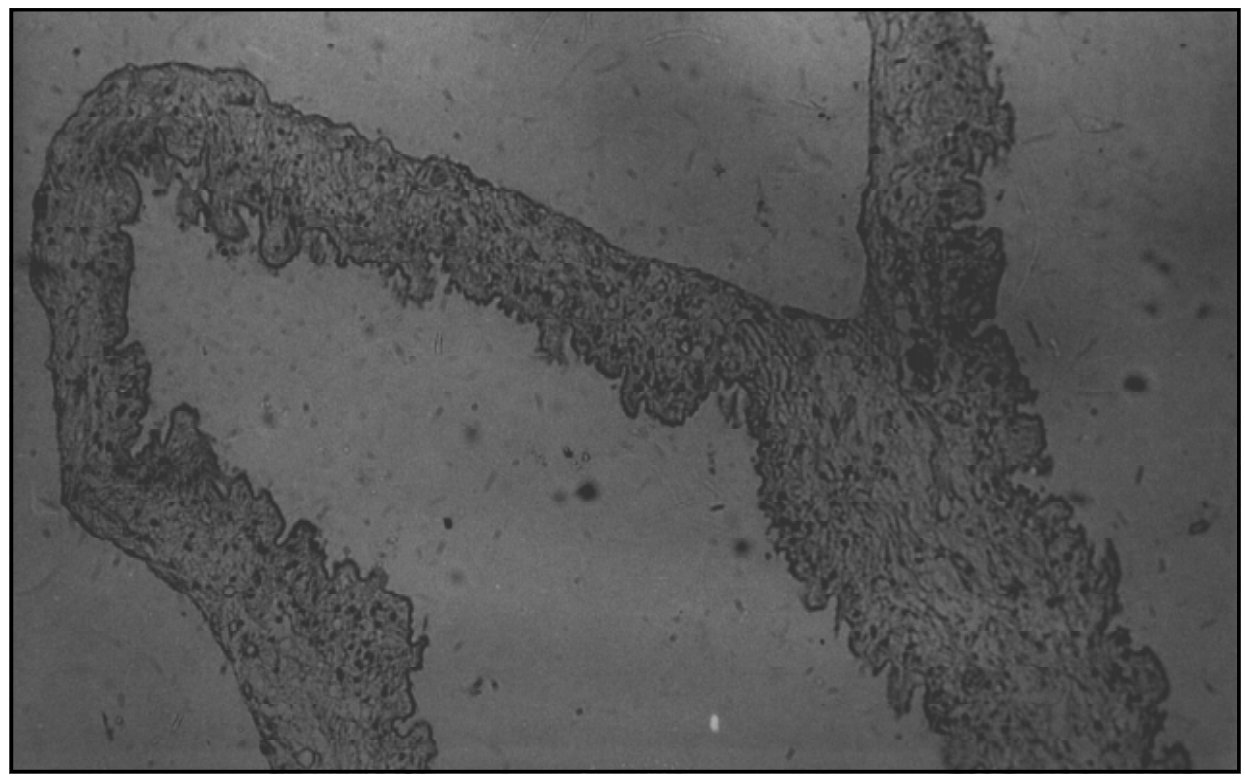

Fig 3. Estudo histológico mostrando membrana envoltória, confirmando tratar-se de cisticerco (HE).

Tabela 1. Revisão dos casos de cisticercose intramedular relatados na literatura ${ }^{3}$.

\begin{tabular}{lclc}
\hline Autor e Ano & N $^{\circ}$ Casos & Autor e Ano & N $^{\circ}$ Casos \\
\hline Rokitanski, 1856 Walton, 1881 & 1 & Pichler, 1900 & 1 \\
Wabraun, 1917 & 1 & Knapp, 1919 Kimpton, 1920 & 1 \\
Salles, 1934 & 1 & Barini, 1954 & 1 \\
Paglioli e Dahne, 1955 & 1 & Costa et al, 1957 & 1 \\
Trelles e Roendenbeck, 1958 & 1 & Cabieses et al, 1959 & 2 \\
Rocca, 1959 & 1 & Dixon e Lipscomb, 1961 & 1 \\
Figueiredo, 1963 & 1 & Granés, 1963 & 1 \\
Reixach-Granes e Becker, 1963 & 1 & Portugal e Oliveira, 1964 & 1 \\
Hesketh, 1965 & 1 & Testa, 1965 & 1 \\
Singh et al,1966 & 1 & Arseni e Danaila, 1967 & 1 \\
Trelles et al, 1968 & 1 & Mehta et al, 1971 & 1 \\
Antoniuk et al, 1974 & 1 & Queiroz et al, 1975 & 1 \\
Garza-Merecado, 1976 & 1 & Natajaran et al, 1978 & 1 \\
Akiguchi et al, 1979 & 1 & Holtzman, 1986 & 1 \\
Siqueira et al, 1987 & 1 & Sharma, 1987 & 1 \\
Bally, 1987 & 2 & Venkartaramana, 1988 & 2 \\
Gallani, 1992 & 1 & Mohanty, 1997 & 6 \\
Dantas e Fagundes-Pereira et al, 1998 & 1 & Corral et al, 1996 & 1 \\
& Total & 45 & \\
\hline
\end{tabular}


As diferentes incidências entre o cérebro e a medula tornam evidente a presença de outros fatores. Dentre esses: o fluxo sanguíneo cerebral é cerca de cem vezes maior que o medular ${ }^{7}$; os vasos da medula têm pequeno calibre e baixa pressão, não favorecendo fenômenos embólicos, e condições de expansibilidade ruins no tecido nervoso da medula quando comparado ao cérebro ${ }^{3}$. Achamos lícito supor que o tamanho dos diferentes segmentos medulares pode também estar relacionado com a incidência, ou seja, quanto maior o segmento maior a incidência, sendo portanto, maior na região torácica, seguida pela região cervical, lombar e sacral, assim como ocorre nas lesões neoplásicas.

Sotelo (1985) comenta que o líquor pode não mostrar alterações, devido ao fato de se tratar de cisticerco inativo ou por não estar em contato com o espaço subaracnoideano ${ }^{2}$. Pode-se evidenciar hiperproteinorraquia, embora seja inespecífica na presença de bloqueio liquórico². O diagnóstico presuntivo pré-operatório é simples quando a lesão está associada à forma cerebral ou leptomeníngea da neurocisticercose. Em casos isolados, o diagnóstico pode ser difícil, pois a lesão assemelha-se aos tumores intramedulares e a imunologia para o cisticerco no sangue ou no líquor é frequentemente negativa ou falso-positiva, não havendo eosinofilorraquia ${ }^{1,3}$. Dos 27 casos revistos por Siqueira et al., $75 \%$ foram diagnosticados no ato cirúrgico, 29,8\% por necropsia e apenas um caso no préoperatório, por se tratar de paciente de área endêmica e apresentar calcificações no músculo peitoral ${ }^{3}$. A lesão restrita a um ou dois segmentos, deve ser considerada como importante característica no diagnóstico diferencial entre $\mathrm{CI}$ e os tumores, tendo em vista que estes geralmente atingem um número maior de segmentos ${ }^{2,11}$.

Há alguns anos, o diagnóstico diferencial entre o tumor e o cisticerco intramedular carecia de significância prática, pois ambas as lesões apresentavam indicação cirúrgica. Entretanto, Corral et al. descreveram o primeiro caso de tratamento conservador, usando albendazol $(15 \mathrm{mg} / \mathrm{Kg} / \mathrm{dia}$ por 75 dias) e dexametazona (12 mg/Kg/dia por mais de 90 dias), com resolução completa da lesão cervical à RNM e recuperação dos déficits motores e sensitivos ${ }^{1}$. Portanto, o diagnóstico pré-operatório de CI pode ser importante, tendo em vista a possibilidade de tratamento medicamentoso ${ }^{1,11-13}$. No futuro, com o avanço da técnicas de neuroimagem, o diagnóstico pré-operatório poderá ser feito mais frequentemente ${ }^{2}$.

Entre 20 casos operados, revistos por Holzman et al, a mortalidade foi de 15\%, a morbidade de $85 \% 9$. Sharma et al. revisaram 20 pacientes submetidos à cirurgia, sendo que três faleceram, cinco não apresentaram melhora, $12(60 \%)$ deambulavam com ou sem auxílio ${ }^{10}$.

Conclui-se portanto que, devido à apresentação clínica pleomórfica, que a forma espinhal da cisticercose deve ser considerada como diagnóstico diferencial de muitas doenças neurológicas, especialmente em pacientes com história de neurocisticercose, em regiões endêmicas, como também em imigrantes, nas áreas não endêmicas. A abordagem cirúrgica de tais lesões deve ser minimamente invasiva, e em casos selecionados deve-se tentar o tratamento clínico, tendo em vista as altas taxas de morbimortalidade.

\section{REFERÊNCIAS}

1. Corral I, Quereda C, Moreno A, et al.. Intramedullary cysticercosis cured with drug treatment. Spine 1996;19:2284-2287.

2. Mohanty A, Venkatrama SK, Das S, Das BS, Rao BR, Vasudev MK. Spinal intramedullary cysticercosis. Neurosurgery 1997;1:82-87.

3. Siqueira MG, Koury LS, Boer CAA, Rezende-Filho CP. Cisticerco solitário intramedular. Arq Bras Neurocirurg 1987;6:133-139.

4. Arruda WO, Ramina R, Pedrozo AA, Meneses MS. Brainstem cysticercosis simulating cystic tumor lesion. Arq Neuropsiquiatr 1994, 52: 431-434.

5. Rossitti SL, Roth-Vargas AA, Moreira ARS, Sperlescu A, Araújo JFM, Balbo RJ. Cisticercose espinhal leptomeníngea pura. Arq Neuropsiquiatr 1990;48:366-370.

6. Livramento JA, Spina-França A. Síndrome do líquido cefalorraquiano na neurocisticercose. Arq Neuropsiquiat 1982;1:59-66.

7. Queiroz LS, Pellegrinni A F ${ }^{\circ}$, Callegaro D, Faria LL. Intramedullary cysticercosis. J Neurol Sci 1975;26:61-70.

8. Sotelo J, Guerrero V, Rubio F. Neurocysticercosis: a new classification based on active and inactive forms. Arch Intern Med 1985;145:442-445.

9. Holtzman RNM, Hughes JEO, Sachdev RK, Jarenwattananon A. Intramedullary cysticercosis. Surg Neurol 1986;26:187-191.

10. Sharma BS, Banerjee AK, Kak UK. Intramedullary spinal cysticercosis. Clin Neurol Neurosurg 1987;89:116-117.

11. Takayanagui OM, Jardim E. Aspectos clínicos da neurocisticercose. Arq Neuropsiquiatr 1983;41:53-63.

12. Minguetti G, Ferreira MVC. Ação de corticóides na fase aguda da neurocisticercose. Arq Neuropsiquiatr 1982;40:77-85.

13. Canelas HM, Ricciardi-Cruz O, Escarlante OAD. Cysticercosis of the nervous system. Arr Neuropsiquiatr 1963;21:77-86. 